\title{
Introduction to the special issue on dynamic networks and knowledge discovery
}

\author{
Céline Rouveirol $^{1}$ • Ruggero G. Pensa ${ }^{2}$. \\ Rushed Kanawati ${ }^{1}$
}

Received: 10 June 2017 / Accepted: 22 June 2017 / Published online: 7 July 2017

(C) The Author(s) 2017

Modeling and analyzing networks is a major emerging topic in different research areas, such as computational biology, social science, document retrieval, etc.

Nowadays, scientific communities have access to very large volumes of networkstructured data, such as social networks, gene/proteins/metabolic networks, sensor networks, and peer-to-peer networks. Often, data is collected at different time points allowing to capture a dynamic trend of the observed network. Consequently, the time component plays a key role in the comprehension of the evolutionary behavior of the studied network (evolution of the network structure and/or of flows within the system). Time can help to determine the real causal relationships within, for instance, gene activations, link creation, and information flow. Handling such data is a major challenge for current research in machine learning and data mining, and it has led to the development of recent innovative techniques that consider complex/multi-level networks, time-evolving graphs, heterogeneous information (nodes and links), requiring scalable algorithms that are able to manage large-scale complex networks.

This special issue is the follow-up of the second Dynamic Networks and Knowledge Discovery workshop (DyNaK) ${ }^{1}$ that was held in conjunction to ECML-PKDD 2014 at Nancy on September 15th 2014. The workshop was motivated by the interest of providing a meeting point for scientists with different backgrounds that were interested in the study of large complex networks and the dynamic aspects of such networks. The workshop attracted 15 submissions out of which 8 papers were accepted.

Building on the success of the DyNaK workshop, an open call for papers was issued for this special issue, focusing on the major topic discussed in the workshop: analyzing, modeling and mining large-scale real networks. More than 50 abstracts were received in a first preliminary step, among which 25 were selected for proceeding to long paper submission. 17 high quality

\footnotetext{
1 http://lipn.univ-paris13.fr/dynak2/.

Céline Rouveirol

celine.rouveirol@lipn.univ-paris13.fr

1 LIPN, CNRS UMR 7030, Université Paris 13, Villetaneuse, France

2 University of Torino, Turin, Italy
} 
papers were received at this second step, each of which was reviewed by three reviewers. Only three contributions were finally selected and are part of this special issue.

In the article Scalable computational techniques for centrality metrics on temporally detailed social network, Gunturi et al. propose a novel computational paradigm called epochpoint based techniques for addressing the non-stationarity challenge of temporally-detailed social networks. Using the concept of epoch-points, they develop a novel algorithm for computing shortest path based centrality metric such as betweenness on a temporally-detailed social network.

In Exceptional contextual subgraph mining, Kaytoue et al. present an approach that finds exceptional contextual subgraphs in dynamic attributed graphs generated from a collection of individual behaviors. Such exceptional subgraphs have an accuracy markedly higher in a specific context than the one obtained in the most general context. They propose an original algorithm that uses sophisticated pruning techniques to restrict the search space of vertices, context refinements, and edges to be considered. The algorithm is assessed on two realworld dynamic datasets, one describing mobility in a public bicycle sharing system and one describing behavioural mobility of e-games players.

Finally, in the article Tiles: an online algorithm for community discovery in dynamic social networks, Rossetti et al. propose an algorithm that extracts overlapping communities and tracks their evolution in time following an online iterative procedure. Their algorithm operates following a domino effect strategy, dynamically recomputing nodes community memberships whenever a new interaction takes place. The computation of network substructures is local and as a consequence, the updating process is efficient, and makes it possible to analyze social networks of the order of millions of nodes.

We would like to warmly thank: the ECML-PKDD 2014 team for making it possible to organize the DyNak workshop, Peter A. Flach, who was a very kind and efficient partner in all the steps of elaborating this special issue and Dragos Margineantu for his involvement in editing this special issue; authors, for the quality of their submissions; all the reviewers without whom this special issue would not have been possible, namely: Luca Aiello, Bettina Berendt, Michele Berlingerio, Bjoern Bringmann, Florence d' Alché-Buc, Jean-Charles Delvenne, Manlio De Domenico, Esther Galbrun, Eric Gaussier, Aristides Gionis, Dino Ienco, Baptiste Jeudy, Kristian Kersting, Ni Lao, Huan Liu, Clémence Magnien, Jérôme Mengin, Rosa Meo, Bivas Mitra, Tsuyoshi Murata, Mirco Nanni, Marc Plantevit, Symeon Papadopoulos, Chedy Raissi, Jan Ramon, Ettore Ritacco, Céline Robardet, Claudio Schifanella, Rossano Schifanella, K. Selçuk Candan, Mahito Sugiyama, Yizhou Sun, Nikolaj Tatti, Gabriele Tolomei, Alessia Visconti, Rongjing Xiang, Xifeng Yan.

We hope that you enjoy this special issue. 